\title{
Research on Three-Phase SVPWM Inverter Based on Double Loop Control of MPPT
}

\author{
Zeng Zhiwei ${ }^{1}$, Long Jiangli ${ }^{2}$ and Hu Mingzhe ${ }^{3, a}$ \\ ${ }^{1,2,3}$ Liupanshui Normal University, Department of Physics and Electronic Science, Key laboratory of \\ optoelectronic information technology, Liupanshui,Guizhou553004
}

Keywords: Inverter; SVPWM; Photovoltaic; MPPT; Simulink

Abstract. Building three-phased inverter mathematics model of voltage vector tracking current controller based on Space Vector Pulse Width Modulation (SVPWM) and a novel current scheme under two phase synchronous rotating frame is presented, which uses grid feed forward controller. At the same time, considering the efficiency of photovoltaic modules, using an improved maximum power tracking algorithm. The simulation results show that the off-grid inverter output current stability, Waveform distortion smaller; and photovoltaic system has good dynamic performance and tracking results. When the load changing, the system can always maintain a constant output voltage.

\section{Introduction}

Under the nervous circumstance in resources of world, and all kinds of energy price surge, the development and use of new energy have become an inevitable tendency in the world today. Solar energy has advantages including pollution-free, unlimited etc. Photovoltaic distributed generator will become more and more important in generation system in the future [1].

The quality of output voltage waveform is a very important index in inverter, the inverter is a indispensable constituent part in photovoltaic power generation technology, in general case, smaller harmonic quantity of inverter output voltage waveform is better. Especially under the situation of non-linear load or a mutation in a load, it is still a hot topic in the research of inverter that how to reduce the harmonic quantity to improve the quality of electric energy at present. If you want to gain a good output of voltage with the shape of sine wave, we usually use the control strategies are SVPWM and the second stage LC filter circuit[2], they have less high harmonic and high voltage utilization and so on.

The emerged of high speed DSP provide the convenience to achieve digital control of inverter .Compared with traditional digital control, beat, repetitive-control and so on[3,4], three-phase inverter which based on SVPWM of Voltage current double loop-closed control of MPPT is more effective to improve the dynamic response and interference rejection ability.

\section{The control strategy of three-phase SVPWM inverter}

Fig. 1 shows the main circuit topology structure of three-phase Independent inverter, DC side include MPPT and Boost translation circuit; power switch devices constitute three-phase PWM inverter ,output pass the smoothing of LC filter which is composed of inductance $\mathrm{L}$ on the side of inverter and filtering capacitor $\mathrm{C}$, and then connect to nonlinear load.

From the figure, $\mathrm{L}$ is output filter inductors, $\mathrm{C}$ is the equivalent of output filter capacitor which connected in $\mathrm{Y}$ connection, $\mathrm{V}$ is the output of inverter before smooth, $i$ is current of filter inductance, $V_{L_{1}}$ is the load voltage, ${ }_{L_{1}}$ is the load current .Setting up the state equation of side circuit of the $\mathrm{T}$ rank of transform, getting the formula Eq. 1: 


$$
\left\{\begin{array}{l}
L \frac{d i}{d t}=V-V_{L_{1}} \\
C \frac{d V_{L_{1}}}{d t}=i-i_{L_{1}}
\end{array}\right.
$$

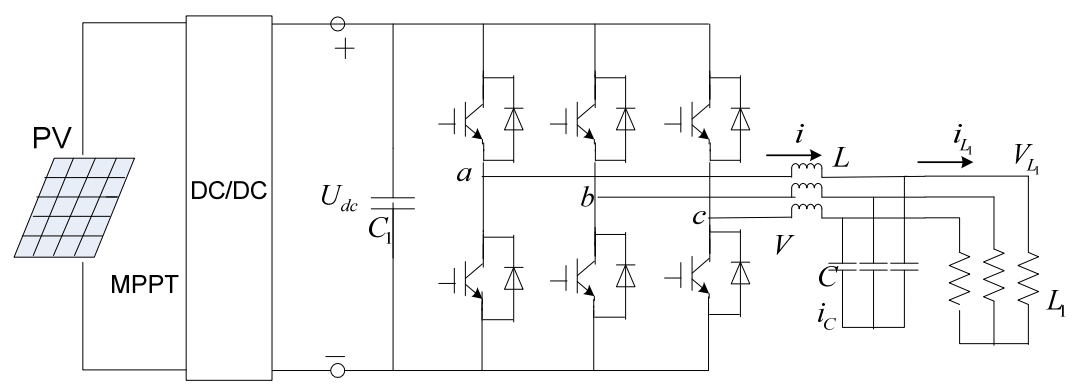

Fig 1. The main circuit topology structure of three-phase independent inverter

In view of the balance of three phase symmetry, and according to the principle that power is not change around the invert[5], using three phase static and two-phase synchronous rotary coordinates, the conversion equation as follows:

$$
\begin{aligned}
& {\left[\begin{array}{l}
u_{\alpha} \\
u_{\beta}
\end{array}\right]=\sqrt{\frac{2}{3}}\left[\begin{array}{rrr}
1 & -\frac{1}{2} & -\frac{1}{2} \\
0 & \frac{\sqrt{3}}{2} & \frac{\sqrt{3}}{2}
\end{array}\right]\left[\begin{array}{l}
u_{a} \\
u_{b} \\
u_{c}
\end{array}\right]} \\
& {\left[\begin{array}{l}
u_{d} \\
u_{q}
\end{array}\right]=\left[\begin{array}{lr}
\cos \omega t & -\sin \omega t \\
-\sin \omega t & \cos \omega t
\end{array}\right]\left[\begin{array}{l}
u_{\alpha} \\
u_{\beta}
\end{array}\right]}
\end{aligned}
$$

Simplifying Eq. 2 and c, we can get the state equations of voltage and current that in the two phase rotating reference frame :

$$
\left\{\begin{array}{l}
L \frac{d i_{d}}{d t}=V_{d}-V_{L_{1} d}+L \omega i_{q} \\
L \frac{d i_{q}}{d t}=V_{q}-V_{L_{1} q}-L \omega i_{d} \\
C \frac{d V_{L_{1} d}}{d t}=i_{d}-i_{L_{1} d}+\omega C V_{L_{1} q} \\
C \frac{d V_{L_{1} q}}{d t}=i_{q}-i_{L_{1} q}+\omega C V_{L_{1} d}
\end{array}\right.
$$

But from Eq. 4, this is a strongly coupled system, the change of current and voltage in axial 'q' will influence the change of current and voltage in axial ' $d$ ', however, the change of current and voltage in axial ' $d$ ' will influence the change of current and voltage in axial ' $q$ ', so, if controlling system included these disturbance quantities, we could nullifying their effect[2]. voltage current two closed loops control system as shown in Fig. 2.

From the off-grid operation control system, we can see that because inverter output side connect load, we can directly control it to outlet constant phase voltage 220V/50HZ. Testing three-phase load voltage to be $V_{L_{1}}$, load current to be $i_{L_{1}}$, the Bidirectional DC-DC now will control DC bus voltage to remain constant. Voltage controller and feedback of voltage output form the voltage outer loop, and 


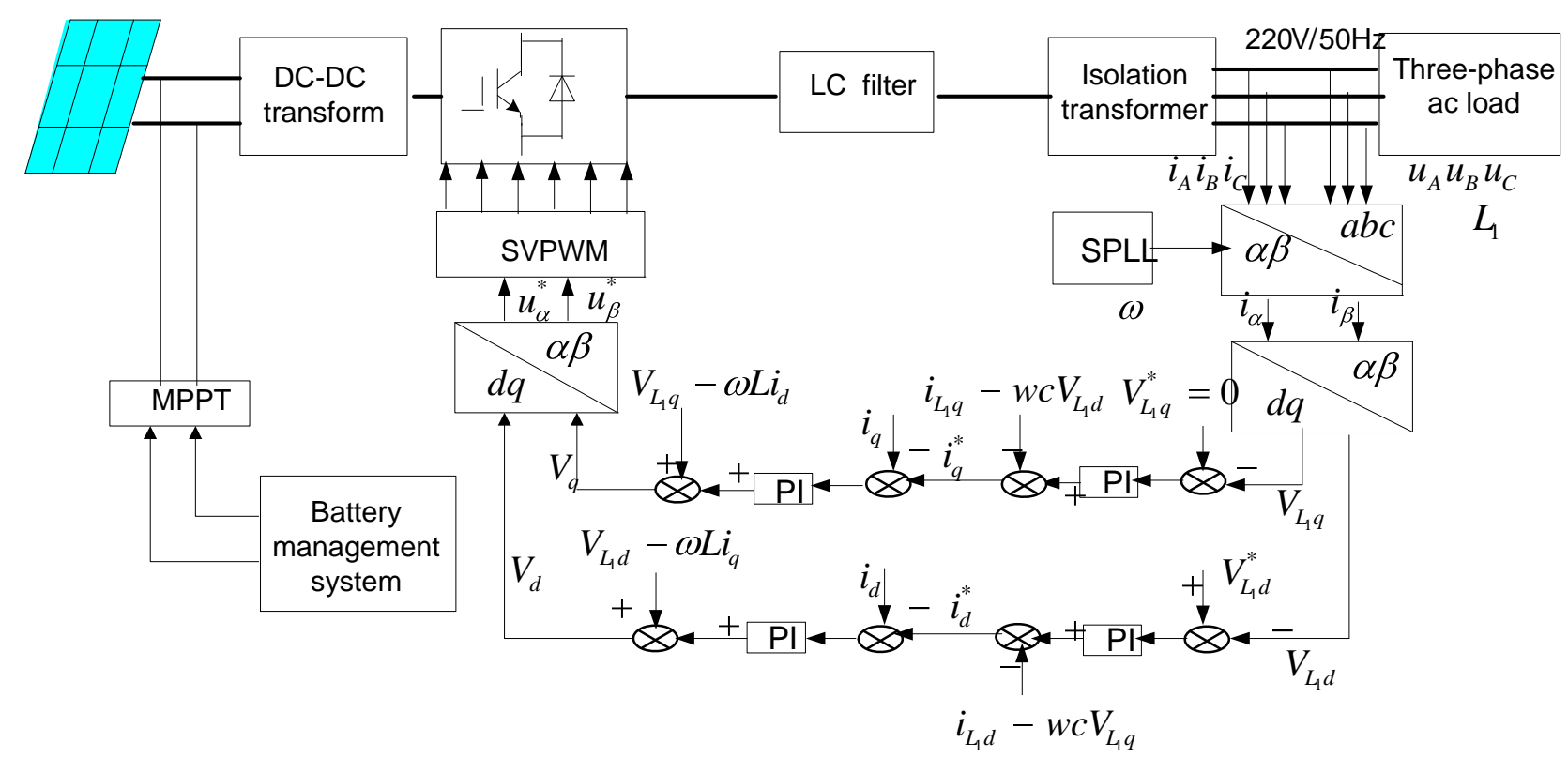

Fig 2.Double-loop Control system of Voltage and current

the current instruction output is to be $i_{d}^{*}$ and $i_{q}^{*}$;Setting $V_{L_{1}}^{*}$ is the maximal value of rated voltage output, then there will exist $V_{L_{1} q}^{*}=0$;Current controller and feedback of current output form the Current Inner Loop ,then its inverter outlet voltage space vector $V_{d}$ and $V_{q}$.In order to increase the system ability of dynamic response and anti-interference, we can introduce load 'current, feed forward of voltage output, capacitance decoupling current and inductance decoupling voltage to it. Finally, using SVPWM control technology adjustment[6] to acquire the driving signal of PWM, and outputting stable sine wave voltage.

\section{MPPT algorithm}

Due to be influenced by external environment, Photovoltaic cell's output voltage always fluctuates and the fluctuation range is large, through optimal control to achieve MPPT, we can get the input reference voltage of photovoltaic power generation system.

At present, the main control methods of MPPT are as follows : Constant Voltage Tracking (CVT)、 Incremental Conductance method (IC) and Perturbation and Observation method (P\&O) [7,8]. CVT is simply and quickly, but it ignores the influence of temperature on photovoltaic battery's output characteristic, the MPPT error is huge; IC is more complex, for it requires a faster speed of control speed, and there is a large amount of data need to be recorded, which increase the requirements of the performance of hardware system as well as the measure accuracy of sensor, thus making the cost increase. But comparing to the above-mentioned two tracking algorithm methods. P\&O is the most adaptable way and the algorithm is easy to realize, but due to the disturbance step should remain unchanged in the process of tracking, which makes the setting value is difficult to be grasped, assuming that the step is set a little big, although it quickly achieve maximum power tracking, It produces power oscillation near the maximum power point; assume that a small step length setting .power oscillation is reduced, but the tracking speed can 't keep up.

To overcome the oscillation in the traditional disturbance observation so as to improve the effect tracking, this text uses the improved perturbation and observation method, which has a better performance, low cost, easy to realize and it's core idea is: in the process of disturbance adoptive change disturbance step constantly, so as to gain higher response speed and steady-state tracking speed.

Due to the sampling time is very short in the process of MPPT ,under this premise can get the slope $\Delta P / \Delta V$ of each working point on the P-V curve.According to the control principle of MPPT as 
shown Fig. 3, when the working point is far from MPP, the bigger the slope, the greater the corresponding $\Delta P / \Delta V$, at this time a large disturbance step can be used; when the working point is

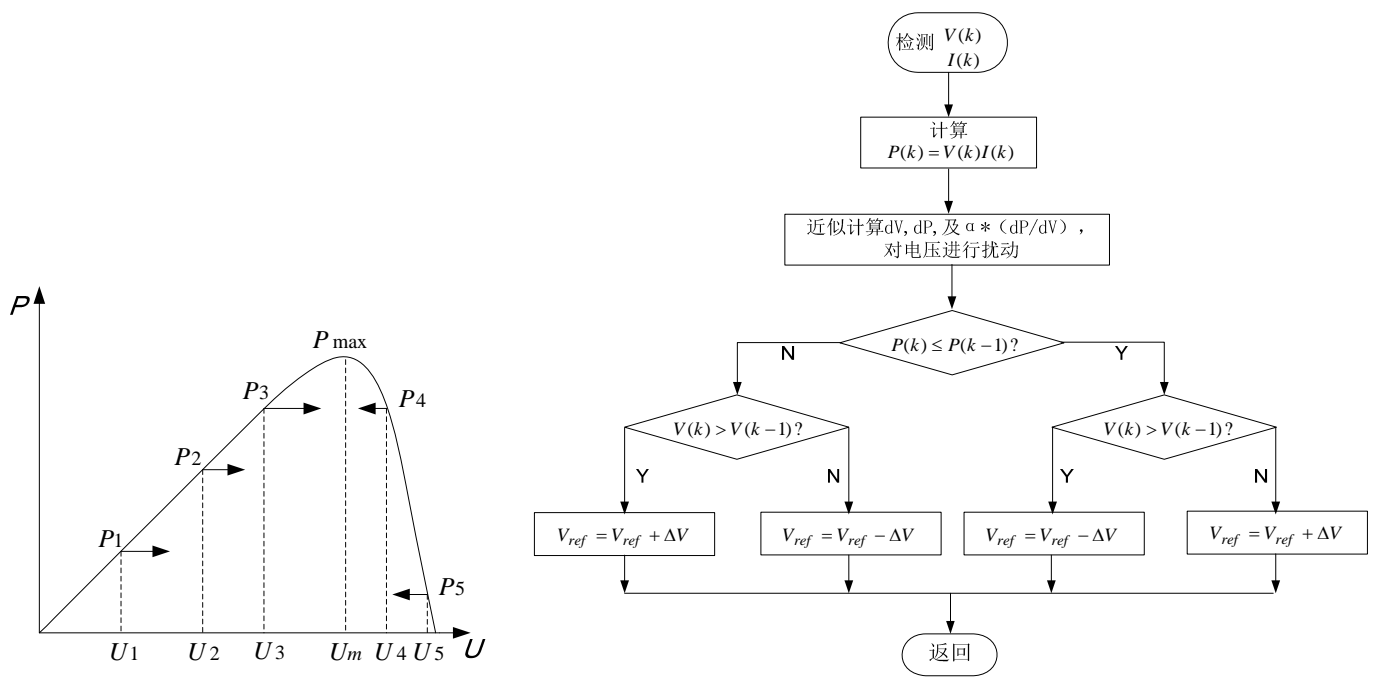

Fig. 3 MPPT control of solar cell Fig. 4 Improved perturbation and observation method flowchart

located in the MPP, the slope is almost close to zero, the equivalent of $\Delta P$ / $\Delta V$ tending to zero, so the small disturbance can be used now, which has a relatively small power oscillation near the MPP. According to the characteristic, adjusted adaptively the perturbation step can improve the tracking speed of the system and reduce the oscillation in the process of tracking, the disturbance step is $\Delta V=\alpha(d P / d V)$, in which ${ }^{\alpha}$ is a positive constant as a speed factor, step speed value is small, before tracking the maximum power, generally there will be a small amount of overshoot Applications according to the actual control requirement and test adjustment to determine the value, the process of improving disturbance observation algorithm is shown Fig. 4.

\section{Simulation results}

Setting the initial conditions: using photovoltaic cells components models for XZSTIl80-24/B, peak power of $180 \mathrm{~W}$, maximum power point voltage is $36 \mathrm{~V}$,maximum working current is $5 \mathrm{~A}$, open circuit voltage of $448 \mathrm{~V}, 5.30 \mathrm{~A}$ short circuit current. $25 \mathrm{KW}$ photovoltaic array, a total of 14 modules in series and 10 in parallel. PV array maximum open-circuit voltage is 627.2, short circuit current is 53A.In the condition $G=1000 \mathrm{~W} / \mathrm{m}^{2}, T=25^{\circ} \mathrm{C}$,.maximum power point of PV array working in voltage $504 \mathrm{~V}$,current $50 \mathrm{~A}$, set $311 \mathrm{v}$ as output phase voltage amplitude and reactive load $\mathrm{L}=5.78 \mathrm{H}$,set up the simulation model; Using Matlab software simulation, the simulation time is $0.2 \mathrm{~s}$, and the result is as follows;
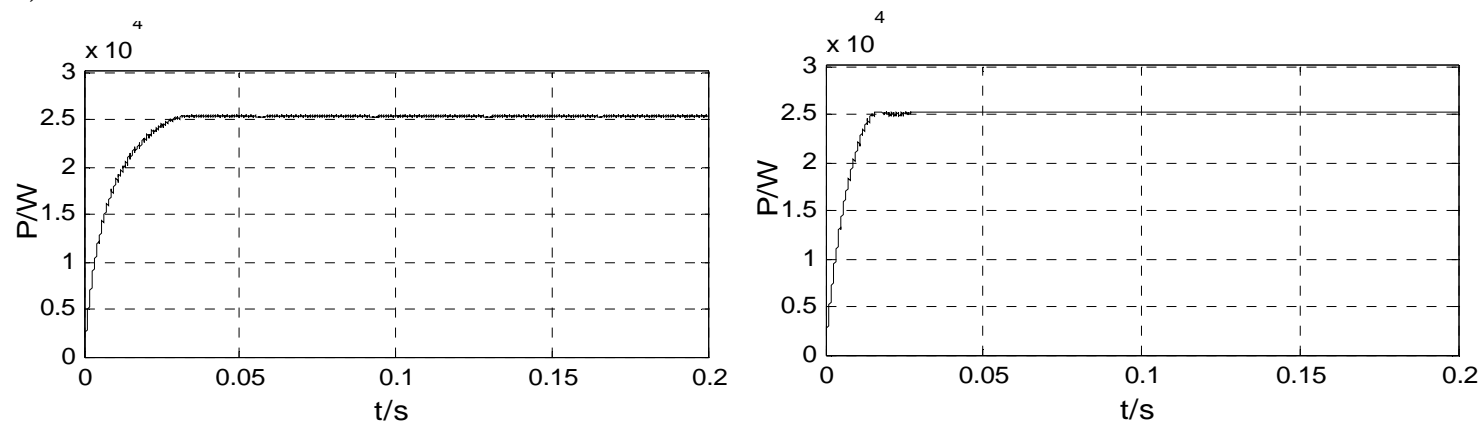

a)

a) 


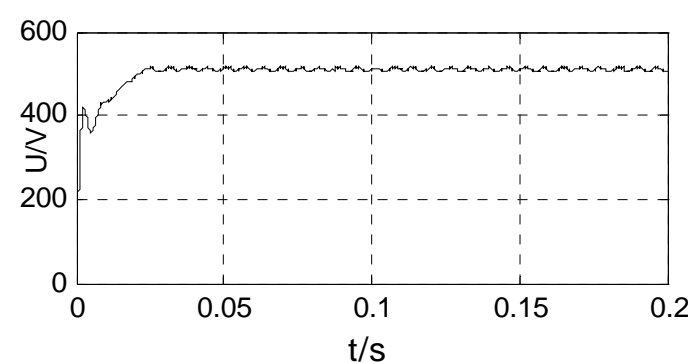

b)

Fig. 5 Output voltage and power waveform

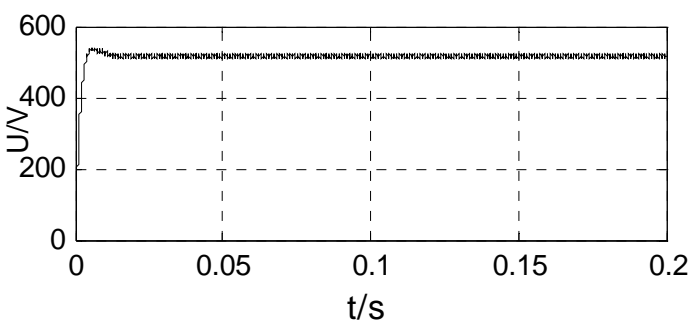

b)

(Traditional perturbation and observation method) (Variable step size perturbation and observation method)

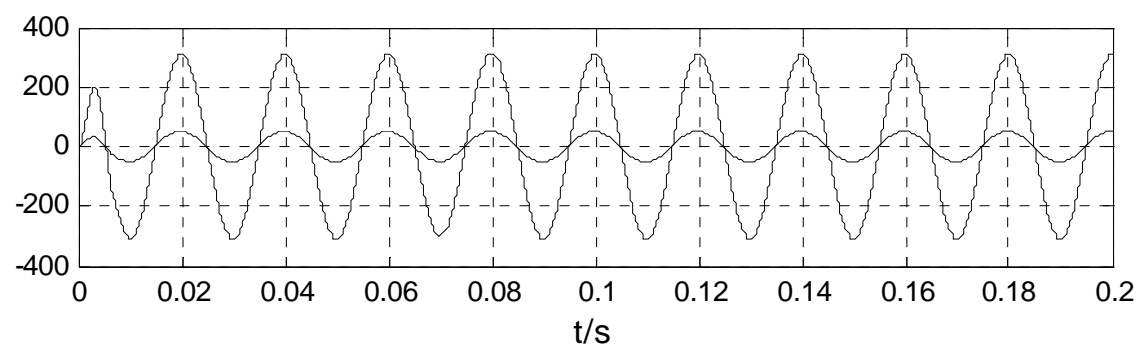

Fig. 7 Voltage and Current Waveforms of Inverter in Standalone Mode
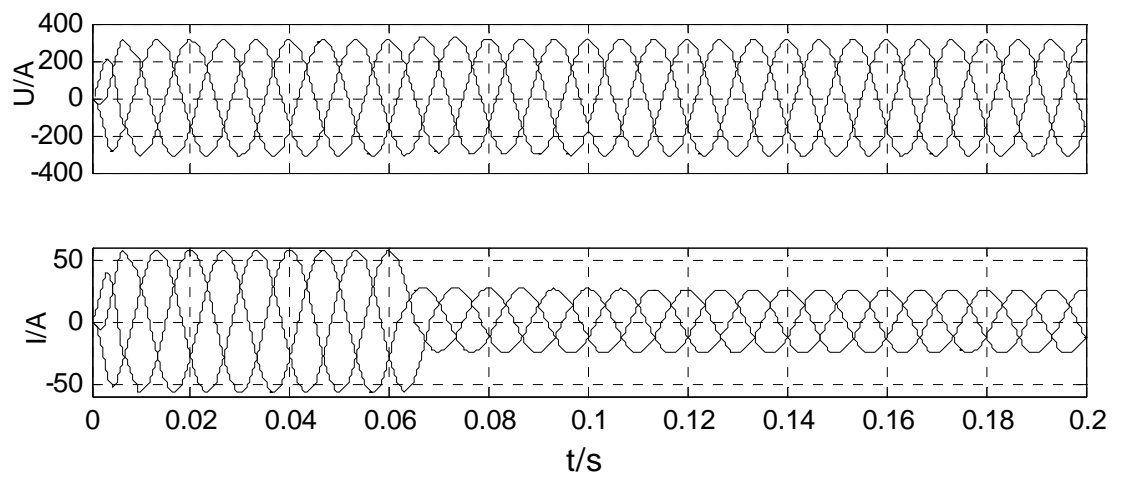

Fig. 8 Voltage and Current Waveforms of Load of Increasing Load in Standalone

Fig. 5 uses the PV array of traditional disturbance observation to output voltage waveform, voltage oscillation is very big during the initial stage when the system is following, the MPP is achieved and stable in $504 \mathrm{~V}$ at around $0.03 \mathrm{~s}$, which accompanied by a large power oscillation. Using the improved disturbance observation as is shown Fig. 6, tracking speed significantly speeding up, the MPP point oscillation decreased significantly and running more stable. Compared with traditional method, this method can more maximum use of solar energy, which verify the feasibility of improved algorithm, and has good tracking performance and stability, Fig. 7 shows that the inverter output current has the same frequency and phase with the network voltage. At same time the power factor is one. And the inverter output voltage and current waveform is very smooth, nearly no rush and burr, which indicates that adopts the capacitor voltage outer ring and the inductance current inner loop control strategy, off-grid inverter output current is more stable and waveform distortion is small, Fig. 8 shows when the system is loaded suddenly at $0.06 \mathrm{~s}$, the load current changes and the load voltage does not change with current and always maintain a constant voltage.

\section{Conclusions}

In combination with MPPT of PV array and SVPWM inverter for double-loop control, studying on key technology of three-phase PV off-grid system, verified that using perturbation steps has the 
advantages of high tracking speed, high precision and little choppy by simulation. On the Off-grid system, introducing the control strategy of voltage current dual-loop, improved the system dynamic response and anti-jamming ability, at the same time, output current of grid-inverter is stable, distortion degree of signal is small. When the load mutation, Current mutation of the load, but Load voltage no change, always maintaining a constant voltage, and it has the reliable electricity for important load.

\section{References}

[1] ZENG Ming, TIAN Guo. Economic Benefits Analysis of Distributed Generation and Its Assessment Model[J]. Power System Technology. 2010.34(8):129-133.

[2] LI Xun, ZHU Peng. Study of the Three-phase SVPWM Inverter based on Double Loop Control[J].Electric power electronic technology.2003.37(5):30-33.

[3] Kukrer O.Deadbeat. Control of a Three-phase Inverter with an Output LC Filter[J].IEEE Trans. On Power Electronics,1996,11(1):16-23.

[4] Jia Yaoqin, Zhu Minglin, Feng Yong.State Feedback Based Repetitive Control for Single-Phase Inverter[J].Transactions of China Electrotechnical Society.2014.29(6):57-63.

[5] WANG Z T, CHANG L C, MAO M Q. DC voltage sensorless control strategy for three-phase grid-connected inverter[A]. Power Electronics Specialists Conference [C]. Rhodes: IEEE, 2008.323-329.

[6] Chang Jun, Ma Shuyun, Zhao Jin.Microcomputer-bused Real-time Modulation Method of Inverter Space Vector Symmetric PWM Signal [J].Electric power electronic technology. 1993.33(6):49-50.

[7] ZHU Tuofei, CHEN Guoding. Survey of MPPT for photovoltaic power generation[J]. Chinese Journal of Power Sources. 2011.35(10):1322-1344.

[8] Wu Junjuan, Jiang Yida.An improved MPPT control algorithm of PV system[J]. Acta Energies Solaris Sinica .2012.33(3):478-483. 